\title{
Éditorial : Les familles et le vieillissement: De troubles privées à un programme mondial
}

Dans sa préface à un livre récent sur l'état des recherches dans les sciences sociales, Newby (2015) nous ramène à la prière de $\mathrm{C}$. Wright Mills d'élever les problèmes privés au niveau des questions publiques. Newby a affirmé que les sociologues, pour longtemps, ont rendu le privé publique en créant «les idées, la perspicacité, la compréhension et, surtout, le débat là où il n'y a pas de solutions simples à nos problèmes privés collectifs» (Newby, 2015, p. viii). Les familles constituent un modèle des défis inhérents à rapprocher [combler] le privé et le publique. Nous «connaissons les familles» dont les membres sont incorporés dans leurs problèmes privés; également, nous nous efforçons de connaître les familles comme chercheurs et influenceurs de leurs personnages publics.

Les articles en ce numéro spécial sur Les familles et le vieillissement dans un contexte mondial reflètent la tradition de soulever les problèmes et de penser critiquement sur les débats. Autour du monde, le discours sur les familles les identifie en tant que le groupe le plus important offrant les relations sociales et de soutien pour les personnes âgées (Keating, 2011). Ce discours a été intensifié face à la pauvreté, les pandémies, et l'évolution des structures et des obligations familiales. Aujourd'hui les familles sont considérées à la fois comme déficientes dans les soins qu'ils dispensent et compromises dans leur capacité à le faire (Uhlenberg et Cheuk, 2008).

Le vieillissement de la population entraîne une grande partie du débat contemporain sur les familles. Les familles sont apparus sur l'ordre du jour public comme un filet de sécurité contre les préoccupations sur la santé, l'économique, et les systèmes de soins sociaux insoutenables. En dépit des preuves de l'écoulement des ressources à travers les générations (Albertini et Kohli, 2013), et des voix apaisantes quant à la viabilité des systèmes de sécurité du revenu (Denton et Spencer, 2011), nous voyons les restes de la démographie apocalyptique (Gee et Gutman, 2000) dans l'ordre du jour des familles. Le renforcement de la centralité de la famille vient des impératifs moraux et parfois juridiques pour soutenir les membres de la famille plus âgés (Haberkern et Szydlik, 2010). Que ce soit basée sur le coût ou la compassion, les familles sont un thème clé dans le vieillissement de la population.
Pourquoi les familles mondiales? Ce numéro spécial a été développé pour capturer discussions à travers les régions du monde sur le rôle des personnes âgées dans les familles. L'impulsion est venue de la conviction que, afin de connaître les familles, nous avons besoin de les comprendre dans leurs diverses formes à travers (et dans) les régions. La gérontologie de la famille a été développée à partir du point de vue de l'hémisphère nord. Pourtant, une perspective élargie sur les familles dans la vie plus tard, révèle divers points de vue sur leur place dans la vie des personnes âgées. Ces perspectives diversement prônent les vertus des grandes familles indépendantes, les familles très unies; signalent d'alarme à propos de la structure de la famille réduite et de sa capacité de prodiguer des soins; et apprécient $l^{\prime}$ intimité à distance. Ces croyances poussent des approches politiques nationales qui ont un impact significatif sur la vie familiale des personnes âgées (L'Initiative mondiale social sur le vieillissement: Rapport sur les activités, 2014). Les articles dans ce numéro spécial représentent certains de ces sensibilités mondiales en Afrique sub-saharienne, l'Australie, l'Europe, la Chine, Israël et les États-Unis. En rendant ce qui est privé publique, ils commencent à cartographier les discours et les réalités de la famille à travers les cultures et les cadres.

\section{Discours sur les obligations familiales}

Les convictions sociales qui concernent la responsabilité des familles pour leurs membres représentent les vues des personnes âgées et du vieillissement de la population. Ces points de vue, à leur tour, reflètent les valeurs sur la manière de répondre aux défis et sur la façon dont les bénéfices ou fardeaux qui découlent devraient être distribués. Van den Broek, Dykstra, et van der Veen abordent la probabilité de tels points de vue étant intégrés dans la vie quotidienne. Ils affirment que les tentatives de changer ou de faire respecter la façon dont une société traite ses membres les plus âgés sont plus susceptibles d'être couronnées de succès si elles ont la plausibilité morale. La plausibilité morale se produit lorsqu'il ya une concordance entre les avertissements de la société et les croyances des citoyens sur la façon dont les charges et les avantages devraient être distribués.

Dans toutes les régions du monde, les discours sociétaux tous montrent la centralité de la famille, mais leurs 
perspectives diffèrent considérablement. En l'Afrique sub-saharienne et en Chine, il y a des commentaires sociétales explicites sur l'importance des familles. En Afrique, ils abordent les forces des grands et forts familles qui s'appuient; en Chine le renforcement des traditions de la piété filiale de longue date, en face de fortes baisses dans la composition générationnelle de la famille. Aboderin et Hoffman disent qu'en Afrique sub-saharienne, la centralité, le caractère unique et l'indispensabilité de «la famille» [(sic)] pour le soutien des membres plus âgés est incontestable. En Chine, les croyances au sujet de la responsabilité de la famille sont également fortes, mais les Chinois sont préoccupés par l'érosion de la piété filiale résultant de la baisse de l'adhésion familiale générationnelle. Phillips et Feng soutiennent, qu'en Chine, l'arrangement idéal pour le soutien familial reste la cohabitation multigénérationnelle. Les familles devront veiller à ce que les personnes âgées reçoivent un soutien financier, des soins de santé, et l'interaction quotidienne avec leurs enfants. Pourtant, les changements dans la composition de la famille et les inégalités croissantes entre les zones rurales et urbaines signifient que ces attentes sont de plus en plus difficile à remplir.

Les discussions des pays plus développés révèlent également les attentes de la responsabilité familiale. Roberto et Blieszner identifient un code idéologique persistante en Amérique du Nord des familles comme nucléaires, très unies, dont les membres résident ensemble, et parfaitement adaptées à prendre soin de leurs membres. Ils affirment que les familles s'infiltrent l'ordre du jour de la politique. Pourtant, la réification de cette structure familiale particulière est en contradiction avec le fait que ces types de familles sont rares aux États-Unis.

Les discours de la famille en Europe et en Australie sont présentés en termes du retrait de l'aide publique pour les soins, ce qui entraîne la formation des familles par défaut. Van den Broek, Dykstra, et van der Veen discutent débats entre les familles et l'État à l'égard de la responsabilité de soigner les personnes âgées. Aux Pays-Bas, du point de vue des membres de la famille, l'ideale pour les soins est d'avoir moins implication de la famille et une plus grande implication de l'État. En revanche, l'État veut une plus grande implication de la famille et la réduction de l'intervention publique. Les auteurs présentent une narration de perte, dans lequel auparavant l'État réduit la pression sur les familles en soutenant les personnes âgées, mais maintenant recule d'une approche du bien-être sociale.

En Australie, la place de la famille dans la lutte contre le vieillissement de la population n'est pas un élément central du débat public. Biggs, Carr et Haapala indiquent que la nécessité de faire face au vieillissement de la population devient accepté en les cercles australiens politiques, mais aucune attention politique se concentre sur le rôle familial des personnes âgées. Plutôt, les discussions politiques australiens sont fondées sur des hypothèses individualistes que les personnes âgées devraient être actives et indépendantes. Biggs et al. décrivent cette approche comme «instrumentalisme économique». Il nie les transitions d'âge légitimes liés au travail, tels que la retraite, tandis que les priorités de développement et de la vie personnelle liées à l'âge risquent d'être ignorées. Ce qui est important du point de vue de l'ordre du jour des familles - ces auteurs suggèrent que cette approche oblige également les gens de prendre un rôle double de l'emploi et des soins qui sont non reconnus. On peut supposer que les adultes plus âgés qui sont actives et indépendantes n'ont pas besoin du soutien des membres de leur famille. Biggs et al. concluent que le décalage entre les initiatives politiques et les priorités familiales et personnelles constitue un nouveau risque social.

Ces discours sur le vieillissement de la population ont apporté des obligations familiales en vue du public. Ils mettent en évidence les croyances au sujet de la forte capacité de prestation de soins de types particuliers de la famille et également leurs déficits de soins. Le discours politique public reflète une évaluation indirecte mais néanmoins puissante des «familles par défaut». À la fois directement et indirectement, ces discours conduisent à la conclusion que, malgré les préoccupations du public au sujet du vieillissement de la population, les soins et le soutien restent des problèmes familiaux privés.

\section{Ressources et contraintes familiales}

Quelles que soient les discours sur l'obligation, les familles sont limitées dans leur capacité ou leur volonté de soutenir les membres les plus âgés. La nature et l'ampleur de ces contraintes sont différentes. Aboderin et Hoffman encadrent leur discussion autour de la vulnérabilité et l'incapacité des personnes âgées résultant de la pauvreté et des situations difficiles de vie. En Afrique sub-saharienne, les personnes âgées peuvent avoir peu d'accès aux soins familiaux à cause de la migration des jeunes vers les zones urbaines et de la pauvreté dans les ménages co-résidents. Dans ces milieux, les soins coulent probablement à travers les générations, en particulier aux petits-enfants vulnérables. La Chine a également connu une réduction de la co-résidence entre les générations. Cong et Silverstein décrivent un modèle de co-résidence contingent, selon lequel les enfants adultes retournent à la maison lorsque les parents sont les plus vulnérables. Ainsi, les enfants modifient mais ne violent pas les obligations relatives à la piété filiale, agissant comme ressources latentes qui sont activés lorsque les besoins des parents deviennent aigus. 
Une volonté contraint d'offrir soins est illustrée dans la recherche de de Jong Gierveld sur les couples plus âgés aux Pays-Bas qui vivaient "séparément ensemble». Environ la moitié des couples VES (vivre ensemble séparément) a déclaré qu'ils ne fourniraient des soins à leurs partenaires, la preuve des engagements contingents entre certains couples à la fin de vie. L'analyse de Roberto et Blieszner des familles pluralistes a également montré les limites des ressources entre les couples. Ceux dont les relations avaient été tendues étaient plus susceptibles d'adopter des comportements potentiellement nuisibles en offrant des soins.

On conteste également les hypothèses monolithiques sur la disponibilité des soins de la famille. Katz, Lowenstein, Halperin et Tur-Sinaï ont examiné les données en provenance d'Europe et d'Israël pour illustrer la variété dans les ressources familiales sur lesquelles les parents et leurs enfants adultes peuvent tirer. Ils ont constaté que le motif le plus fréquent était «familialisme descendant», selon lequel le soutien s'écoule des parents plus âgés à leurs enfants adultes. «Familialisme ascendant» a été associé avec les parents d'âge plus avancé, des problèmes de santé, et avec des niveaux plus élevés de ressources entre les enfants adultes. Près d'un tiers des familles ont été caractérisées par des modèles autonomes de la vie à une distance et les échanges de soutien minime. Roberto et Blieszner défient également les chercheurs à regarder au-delà des hypothèses statiques sur les sources de soins. Ils se demandent si avoir plus d'enfants adultes est une bonne assurance pour la prestation de soins, car ils affirment qu'il ya peu de littérature empirique qui évalue la valeur ajoutée d'avoir plusieurs enfants qui peuvent fournir des soins au lieu d'avoir un seul fournisseur de soins primaires.

\section{Modification des limites de ce qui constitue une famille}

Un des principes de la discussion «de la capacité familiale en déclin» est que les prochaines cohortes des personnes âgées auront moins de possibilités de soutien en raison de la baisse des taux de natalité (Carrière, Keefe, Légaré, Lin, et Rowe, 2007). Cette préoccupation se concentre sur la diminution de la famille nucléaire et surtout les petits nombres d'enfants adultes. Des preuves de la recherche empirique sur la prestation de soins indiquant que les enfants adultes sont le plus grand groupe de soignants (Sinha, 2013) suggère que, dans le nord mondiale, la famille étroitement bornée est une mise au point appropriée des préoccupations quant à la capacité de soutien de la famille. Pourtant, les modèles pluralistes de la vie familiale sont de plus en plus commun. En référence aux États-Unis, Roberto et Blieszner affirment que la flexibilité structurelle est probablement une caractéristique plus commune des familles que les chercheurs ont reconnu. Ils déclarent que nous devons regarder au-delà des conjoints et des enfants adultes à voir, par exemple, si les familles «perches» multigénérationnelles ont une capacité renforcée de se soutenir mutuellement; dans quelles circonstances les beaux-parents sont intégrés comme membres de la famille; et comment les individus GLBT (gais, lesbiennes, bisexuels et transgenres) créent familles. Notre lentille globale suggère qu'un débat plus expansif concernant les limites de la famille est justifié. En Chine, comment violent les normes de la co-résidence générationelle, la co-residence retardée ou différée des enfants adultes et leurs parents vieillissants (Phillips et Feng; Cong et Silverstein)? En Europe, les nouvelles formes de relations entre les couples, comme vivant ensemble séparément, influencent-elles les normes de l'intimité et l'engagement au sein des couples et de leurs réseaux de parenté (De Jong Gierveld)? Quelles sont les frontières autour de familles africaines, quand il est question de l'engagement et des flux de support (Aboderin et Hoffman)? À travers le monde, est-ce-que «faire une famille» assure l'intégration des membres plus âgés?

\section{Ordre du jour mondial pour la recherche sur les familles}

Collectivement, les auteurs de Les familles et le vieillissement dans un contexte mondial ont créé une critique des perspectives sociétales sur les familles des personnes âgées; ont présenté les conclusions empiriques sur les ressources de la famille, les contraintes et les flux de ressources entre les membres des familles; et ont défendu la nécessité de repenser les limites de ce qui fait une famille. Tous ensemble, leur travail ajoute beaucoup à la façon dont nous comprenons les familles. Pourtant, comme les sociologues, notre tâche reste incomplète sans la création d'une base de connaissances. Comme déclarent Maclean et Roberts (2015, p. 128): «Le travail contemporain dans les sciences sociales a une rôle supplémentaire, peut-être encore plus important, à jouer dans la recherche - non seulement pour trouver des réponses aux problèmes connus, mais en continuant développer la prochaine série de questions.»

Beaucoup de questions sont posées par les auteurs dont les articles sont inclus dans ce numéro. Pour les organiser en un programme de recherche est intimidant. Cependant, nous croyons que les thèmes pluridisciplinaires pour un programme de recherche sur les familles des personnes âgées en Afrique du Aboderin et Hoffman intègrent plusieurs questions fondamentales et fonctionneraient bien dans toutes les régions du monde. Ils proposent quatre perspectives pluridisciplinaires 
pour informer l'enquête sur les réalités du familles vieillissantes:

- Une approche interprétative qui remet en question les croyances au sujet de la structure et la qualité familiale.

- L'engagement critique avec les idées théoriques ou conceptuelles saillants dans la littérature gérontologique globale sur les liens familiaux pendant la vie plus tard.

- L'intégration des expériences hétérogènes de familles vieillissantes.

- Mise en place de l'expérience des familles de personnes âgées dans le contexte des tendances mondiales, telles que l'élargissement de la migration et l'accroissement des inégalités dans et entre les pays.

Les familles et le vieillissement dans un contexte mondial est donné aux chercheurs, décideurs et praticiens qui poseront les questions critiques et approfondiront les connaissances. Puissiez-vous améliorer la manière dont nous connaissons les familles, de façon à développer une meilleure qualité de vie pour les personnes âgées et pour la variété de familles dans lesquelles ils vivent leur vie.

Norah Keating and Jenny De Jong Gierveld

Éditeur invité

\section{References}

Albertini, M., \& Kohli, M. (2013). The generational contract in the family: An analysis of transfer regimes in Europe. European Sociological Review, 29(4), 828-840.

Carrière, Y., Keefe, J., Légaré, J., Lin, X., \& Rowe, G. (2007). Population aging and immediate family composition: Implications for future home care services. Genus, LXIII(1), 11-31.
Denton, F. T., \& Spencer, B. G. (2011). Age of pension eligibility, gains in life expectancy, and social policy. Canadian Public Policy, 37, 183-200.

Gee, E. M., \& Gutman, G. M. (Eds.). (2000). The overselling of population aging: Apocalyptic demography, intergenerational challenges, and social policy. Toronto, ON: Oxford University Press.

Haberkern, K., \& Szydlik, M. (2010). State care provision, societal opinion and children's care of older parents in 11 European countries. Ageing and Society, 30(2), 299-323.

Keating, N. (2011). Critical reflections on families of older adults. Advances in Gerontology, 24(2), 343-349.

Maclean, M., \& Roberts, C. (2015). Numbers and questions: The contribution of social science to understanding the family, marriage and divorce. In J. Michie \& C. L. Cooper (Eds.), Why the Social Sciences Matter (pp. 128-142). Basingstoke, UK: Palgrave Macmillan.

Newby, H. (2015). Foreword. In J. Michie \& C. L. Cooper (Eds.), Why the Social Sciences Matter (pp. vii-iix). Basingstoke, UK: Palgrave Macmillan.

Sinha, M. (2013). Spotlight on Canadians: Results from the General Social Survey. Statistics Canada, Catalogue no. 89-652-X No. 001.

The Global Social Initiative on Ageing Report on Activities (2014). Downloaded March 11, 2015 from http://wun.ac.uk/ files/2014jun3_gsia_2013_final_report.pdf.

Uhlenberg, P., \& Cheuk, M. (2008). Demographic change and the future of informal caregiving. In M. Szinovacs \& A. Davey (Eds.), Caregiving contexts. Cultural, familial and societal implications (pp. 9-33). New York, NY: Springer. 